\title{
Novel Curcumin C66 That Protects Diabetes-Induced Aortic Damage Was Associated with Suppressing JNK2 and Upregulating Nrf2 Expression and Function
}

\author{
Cheng Li $\left(\mathbb{D},{ }^{1}\right.$ Xiao Miao $\left(\mathbb{D},{ }^{2}\right.$ Shudong Wang $\left(\mathbb{D},{ }^{1}\right.$ Binay Kumar Adhikari, ${ }^{1}$ Xin Wang $\mathbb{D}^{3},{ }^{3}$

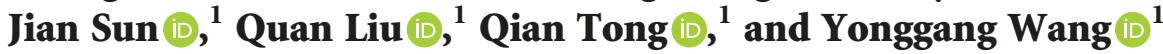 \\ ${ }^{1}$ Department of Cardiovascular Center, The First Hospital of Jilin University, Changchun, Jilin 130021, China \\ ${ }^{2}$ The Second Hospital of Jilin University, Changchun, Jilin 130000, China \\ ${ }^{3}$ Department of Thyroid Surgery, The First Hospital of Jilin University, Changchun, Jilin 130021, China
}

Correspondence should be addressed to Qian Tong; tongqian187@aliyun.com and Yonggang Wang; xiaogang94@163.com

Received 15 July 2018; Revised 23 September 2018; Accepted 10 October 2018; Published 28 November 2018

Guest Editor: Ayman M. Mahmoud

Copyright ( $\odot 2018$ Cheng Li et al. This is an open access article distributed under the Creative Commons Attribution License, which permits unrestricted use, distribution, and reproduction in any medium, provided the original work is properly cited.

\begin{abstract}
Diabetes-related cardiovascular diseases are leading causes of the mortality worldwide. Our previous study has explored the protective effect of curcumin analogue C66 on diabetes-induced pathogenic changes of the aorta. In the present study, we sought to reveal the underlying protective mechanisms of C66. Diabetes was induced in male WT and JNK2 ${ }^{-l-}$ mice with a single intraperitoneal injection of streptozotocin. Diabetic mice and age-matched nondiabetic mice were randomly treated with either vehicle (WT, WT DM, JNK2 $2^{-1-}$, and JNK2 $\left.{ }^{-1-} \mathrm{DM}\right)$ or C66 (WT + C66, WT DM + C66, JNK2 $2^{-1-}+\mathrm{C} 66$, and JNK2 $2^{-1-} \mathrm{DM}$ + C66) for three months. Aortic oxidative stress, cell apoptosis, inflammatory changes, fibrosis, and Nrf2 expression and function were assessed by immunohistochemical staining for the protein level and real-time PCR method for mRNA level. The results suggested that either C66 treatment or JNK2 deletion can reverse diabetes-induced aortic oxidative stress, cell apoptosis, inflammation, and fibrosis. Nrf2 was also found to be activated either by C66 or JNK2 deletion. However, C66 had no extra effect on diabetic aortic damage or Nrf2 activation without JNK2. These results suggest that diabetes-induced pathological changes in the aorta can be protected by C66 mainly via inhibition of JNK2 and accompanied by the upregulation of Nrf2 expression and function.
\end{abstract}

\section{Introduction}

Cardiovascular diseases are associated with a substantial morbidity and mortality worldwide. People with diabetes mellitus exhibit a higher risk of cardiovascular diseases compared with that of the general population. Cardiovascular complications caused more than a half of death in diabetic patients $[1,2]$. There is a prediction that the number of diabetic patients reaches almost 600 million by 2035 [3]. Hyperglycemia causes irreversible damage to blood vessels by inducing both micro- and macrovascular complications in various organs like the skin, muscles, heart, brain, eyes, and kidneys $[4,5]$. Poor control of blood glucose at the early stage of diabetes has been demonstrated to accelerate the incidence and progression of vascular damage. Therefore, new therapies to prevent diabetic complications should be paid more attention.

There is considerable evidence that hyperglycemiainduced vascular damage is associated with the generation and accumulation of reactive oxygen species, ultimately leading to increased oxidative stress $[6,7]$. Oxidative stress exhibits an imbalance between the free radical production and the endogenous physiological antioxidant mechanisms that lead to the activation of stress-sensitive intracellular signaling pathways and increased cellular damage. The damage of endothelial cells contributes to vasoconstriction, cellular proliferation, leukocyte aggregation, thrombosis, and inflammation predisposing to atherosclerosis [8]. It has been reported that the lipid bilayer of endothelia cells can be destroyed by reactive oxygen species, releasing inflammatory 
and apoptotic cytokines [9]. Moreover, injury to endothelial cells causes collagen exposure, platelet activation, and aggregation at the injury site, thus contributes to a cascade of thrombosis and inflammation [10]. It is believed that oxidative stress and inflammation are reciprocal causes and outcomes. Nuclear factor (erythroid-derived 2)-like 2 (Nrf2) plays a crucial role in the regulation of the environmental stress by inducing the expression of detoxification and antioxidant enzymes. Under unstressed condition, Nrf2 can be ubiquitinated and degraded by its inhibitor Kelch-like ECH-associated protein 1 (Keap1). When cells are exposed to oxidative stress, Nrf2 can dissociate from Keap1 and translocate to the nucleus, leading to its activation [7]. Once Nrf2 is activated, it regulates the expression of a vast number of genes, including those genes that regulate antioxidants and detoxification enzymes as well as inflammatory responses [11]. It has been shown that the activation of Nrf2 can reduce oxidative stress and inflammation in diabetes, while its absence can aggravate the diabetic complications $[12,13]$. Therefore, the activation of Nrf2 may be particularly helpful in combating the deleterious effects of hyperglycemic stress.

The c-Jun N-terminal kinases (JNKs) can be activated by a range of stimuli and were known as "stress-activated protein kinases". JNKs, belonging to the mitogen-activated protein kinase superfamily, play a crucial role in stress responses, cell survival, and apoptosis [14]. There are three isoforms: JNK1, JNK2, and JNK3; JNK1 and JNK2 are ubiquitously expressed, and JNK3 expresses strictly in the brain, heart, and testis [15].

It has been reported that JNK2 is associated with hypercholesterolemia-induced endothelial dysfunction and oxidative stress and is required for foam cell formation within the atherosclerotic plaque $[16,17]$. And JNK2 isoform has shown a more prominent role in the development of obesity-associated insulin resistance [18]. Moreover, deletion of JNK2 has been demonstrated to block diabetic-induced protein nitroxylation [19]. Thus, we speculate that JNK2 plays an important role in diabetes-induced aortic damage.

Curcumin, a natural compound, is the most active agent of the polyphenolic curcuminoids derived from the root of turmeric (Curcuma longa). Traditionally, it has been widely used as an herbal medicine and/or food flavoring. Recently, compelling studies show the protective effect of curcumin on human health through its anti-inflammatory, antioxidant, and antimicrobial properties [20-22]. Therefore, curcumin and its analogues have attracted extensive attention. Several studies have reported that (2E,6E)-2,6bis[2-(trifluoromethyl)benzylidene]cyclohexanone (so-called compound C66), a novel curcumin, has memorable effects in diabetes-related complications based on its anti-inflammatory, antifibrotic, antioxidative, and antiapoptotic properties [23-25]. These studies have also demonstrated that the C66 protection in diabetes is accompanied by inhibition of JNK function. A molecular docking predicted that C66 may target JNK2, which leads to its protective properties [26]. Therefore, we use JNK2 gene knockout mice to verify that the protection of C66 on diabetes-induced aortic damage is associated with inhibition of JNK2.

\section{Material and Methods}

2.1. Animals. $\mathrm{JNK}^{-1-}$ and wild-type (WT) male mice on B6.129S2-Mapk9tm1Flv/J genetic background, 6-8 weeks of age, were purchased from the Jackson Laboratory (Bar Harbor, ME, USA). Animals were housed in the Animal Center of Jilin University at a constant room temperature with a 12:12 light-dark cycle. A standard rodent diet and water were provided. All animals were acclimatized to the environment for 1 week before being used. Type 1 diabetic mouse model was established by intraperitoneal injection of STZ ((Sigma-Aldrich, St. Louis, MO, USA), dissolved in $0.1 \mathrm{M}$ sodium citrate buffer ( $\mathrm{pH} 4.5)$ ) at $150 \mathrm{mg} / \mathrm{kg}$, while the control animals received the same volume of sodium citrate buffer. Three days after STZ injection, the blood glucose was tested by a glucometer, and the blood glucose levels $\geq 250 \mathrm{mg} / \mathrm{dl}$ were considered as diabetic (DM). Then, both diabetic WT mice and JNK2 ${ }^{-1-}$ mice were randomly divided into two groups: WT DM $(n=8)$ and C66-treated WT DM (WT DM + C66, $n=8)$ and JNK2 ${ }^{-1-}$ DM $(n=8)$ and C66-treated $\mathrm{JNK}^{-1-} \mathrm{DM}\left(\mathrm{JNK}^{-/-} \mathrm{DM}+\mathrm{C} 66, n=8\right)$. The age-matched control WT and JNK2 ${ }^{-l-}$ mice were also randomly divided into two groups, respectively: WT $(n=8)$ and C66-treated WT (WT + C66, $n=8)$ and JNK2 ${ }^{-1-}(n=8)$ and C66-treated JNK2 $2^{-1-}\left(\mathrm{JNK}^{-1-}+\mathrm{C} 66, n=8\right)$. In the four C66-treated groups, mice were orally administered C66 at $5 \mathrm{mg} / \mathrm{kg}$ once a day in alternating days for 3 months, while the four matched groups were given 1\% CMC-Na solution alone according to the same schedule.

2.2. Aorta Preparation and Histology Staining. Animals were executed after anesthesia, and the thoracic aortas were isolated. Aortic tissues were fixed in $4 \%$ paraformaldehyde for more than $24 \mathrm{~h}$, and then they were dehydrated and paraffin-embedded. The fixed tissues were cut into $4 \mu \mathrm{m}$ thick sections for Masson's trichrome staining and immunohistochemical staining.

For immunohistochemical staining, the tissue slices were deparaffinized by dimethylbenzene, dehydrated by graded ethanol, and then microwaved for $10 \mathrm{~min}$ in $1 \%$ PBS buffer ( $\mathrm{pH}$ 7.4, Sangon Biotech Inc., Shanghai, China) for antigen retrieval. When the tissue slices were cooled at room temperature, they were washed with $1 \%$ PBS three times and infiltrated with $0.1 \%$ Triton X-100 for $15 \mathrm{~min}$. In order to block endogenous peroxidase, all tissue slices were incubated with $3 \%$ hydrogen peroxide for $10 \mathrm{~min}$ in the dark. The tissue slices were incubated with $10 \%$ goat serum for $60 \mathrm{~min}$ at $37^{\circ} \mathrm{C}$ and then with primary antibodies (anti-MCP-1 $1: 250$, anti-TNF- $\alpha 1: 300$, anti-CTGF $1: 150$, anti-TGF- $\beta 11: 200$, anti-HO-1 1:150, and anti-SOD-1 1:50 (Abcam Inc., America)) at $4^{\circ} \mathrm{C}$ overnight. Thereafter, all sections were incubated with secondary antibodies (HRP-labeled goat anti-rabbit IgG $(\mathrm{H}+\mathrm{L})$ 1:400 or HRP-labeled goat antimouse IgG $(\mathrm{H}+\mathrm{L}) 1: 400$ (Beyotime Inc., Shanghai, China)) for $40 \mathrm{~min}$ at $37^{\circ} \mathrm{C}$ and then stained with $\mathrm{DAB}$.

For immunofluorescent staining, after antigen retrieval and $0.1 \%$ Triton X-100 infiltration, primary antibody anti-Nrf2 was used at $4^{\circ} \mathrm{C}$ overnight. Thereafter, all sections were incubated with secondary antibody (Cy3-labeled 
Table 1: Primer sequences for real-time quantitative PCR.

\begin{tabular}{lrc}
\hline Gene & Forward primer & Reverse primer \\
\hline CTGF & GGGCCTCTTCTGCGATTTC & ATCCAGGCAAGTGCATTGGTA \\
TGF- $\beta 1$ & CTCCCGTGGCTTCTAGTGC & GCCTTAGTTTGGACAGGATCTG \\
MCP- 1 & TTAAAAACCTGGATCGGAACCAA & GCATTAGCTTCAGATTTACGGGT \\
TNF- $\alpha$ & CCCTCACACTCAGATCATCTTCT & GCTACGACGTGGGCTACAG \\
HO- 1 & AAGCCGAGAATGCTGAGTTCA & GCCGTGTAGATATGGTACAAGGA \\
SOD- 1 & AACCAGTTGTGTTGTCAGGAC & CCACCATGTTTCTTAGAGTGAGG \\
Nrf 2 & CTTTAGTCAGCGACAGAAGGAC & AGGCATCTTGTTTGGGAATGTG \\
\hline
\end{tabular}

goat anti-rabbit IgG $(\mathrm{H}+\mathrm{L})$ (Beyotime Inc., Shanghai, China)) for $40 \mathrm{~min}$ at $37^{\circ} \mathrm{C}$ and then stained with DAPI. A negative control was performed just by incubating with secondary antibody.

2.3. Terminal Deoxynucleotidyl Transferase-Mediated dUTP Nick End Labelling (TUNEL) Staining. TUNEL staining was performed with formalin-fixed and paraffin-embedded sections using TUNEL staining kit (DeadEnd ${ }^{\mathrm{TM}}$ Colorimetric TUNEL System, Promega Inc., USA) according to the manufacturer's instructions. Positively stained apoptosis cells were counted in at least five random microscopic fields for each slice. Each group had five slices. Cells with TUNEL-positive nuclei were counted under high magnification 400x. The results were presented as TUNEL-positive nuclei per 100 vascular cell nuclei.

2.4. Quantitative Real-Time PCR ( $q R T-P C R)$. Total RNA was extracted from aortic tissues using the AxyPrep ${ }^{\mathrm{TM}}$ multisource total RNA kit (Axygen Scientific Inc.). RNA was reverse transcribed to cDNA using the TransScript All-inOne First-Strand cDNA Synthesis SuperMix (Transgen Biotech Inc., Beijing, China). Real-time quantitative RTPCR analysis was carried out using the TransStart Top Green qPCR SuperMix (Transgen Biotech Inc., Beijing, China) and the ABI 7300 Real-Time qPCR System. The primers of CTGF, TGF- $\beta 1$, MCP- 1 , TNF- $\alpha$, HO- 1 , SOD- 1 , and Nrf2 were synthesized by Sangon Biotech (Shanghai, China), and the sequences are listed in Table 1. Data were expressed as number of fold increase compared with levels measured in controls by using the $\Delta \Delta^{\mathrm{Ct}}$ method and $\beta$-actin as a reference gene.

2.5. Statistical Analysis. Variation between different groups was analyzed by one-way analysis of variance (ANOVA) test using Tukey test with Origin 8.0 Lab data analysis. Statistical significance was considered if $P<0.05$.

\section{Results}

3.1. Either JNK2 Deletion or C66 Treatment Can Attenuate Diabetes-Induced Aortic Fibrosis. At the end of the experiment, the collagen accumulation in tunica media of the aortas was examined by Masson staining (Figure 1). The results showed that C66 treatment or JNK2 deletion can significantly reverse collagen accumulation in the aortas in diabetic mice. However, there was no significant difference between the DM group and DM + C66 group in $\mathrm{JNK}^{-/-}$mice.

Immunohistochemical stain was used to evaluate the expression of profibrotic mediators, CTGF (Figure 2(a)) and TGF- $\beta 1$ (Figure 2(d)) in aortic tunica media. Supplementation with $\mathrm{C} 66$ or deletion of JNK2 obviously prevented these fibrotic responses in the aortas of diabetic mice (the WT DM + C66 group and $\mathrm{JNK} 2^{-/-} \mathrm{DM}$ group) (Figures 2(b) and 2(e)). Similarly, C66 has not shown its further effect in $\mathrm{JNK}^{-/-} \mathrm{DM}$ mice. We also used qPCR to evaluate the mRNA levels of CTGF (Figure 2(c)) and TGF- $\beta 1$ (Figure 2(f)). The results were consistent with the immunohistochemical stain.

\subsection{Either JNK2 Deletion or C66 Treatment Can Attenuate} Diabetes-Induced Aortic Cell Apoptosis. We used the TUNEL assay to analyze apoptosis of aortic cells (Figure 3(a)). The results showed that diabetes-induced increased apoptosis can be significantly reversed by C66 treatment or JNK2 deletion, but $\mathrm{C} 66$ seemed to have no effect on JNK2 deletion diabetic mice (Figure 3(b)). Similar result was also found in the mRNA expression of apoptosis-related protein caspase- 3 (Figure 3(c)) via qPCR.

\subsection{Either JNK2 Deletion or C66 Treatment Can Attenuate} Diabetes-Induced Aortic Inflammation and Oxidative Stress. On account of the fact that both inflammation and oxidative damage are primary risk factors for the vascular endothelial remodeling, the expression of TNF- $\alpha$ (Figure 4(a)) and MCP-1 (Figure 4(d)) was examined with immunohistochemical staining, which showed a significant increase in aortic tunica media of diabetic mice, an effect that was completely prevented by C66 treatment or JNK2 deletion. Additionally, C66 was found to have no special effects on these inflammatory factors in JNK2 ${ }^{-/-}$DM mice (Figures $4(\mathrm{~b})$ and 4(e)). The results of qPCR of TNF- $\alpha$ (Figure 4(c)) and MCP-1 (Figure $4(\mathrm{f})$ ) were consistent with immunohistochemical staining.

Considering that inflammation and oxidative stress are reciprocal cause and outcomes, 3-NT (Figure 5(a)) and 4-HNE (Figure 5(b)) were examined by immunohistochemical staining to evaluate oxidative and nitrative damage. The elevation of 3-NT and 4-HNE in the diabetic aorta can be significantly decreased by C66 or JNK2 deletion. However, 

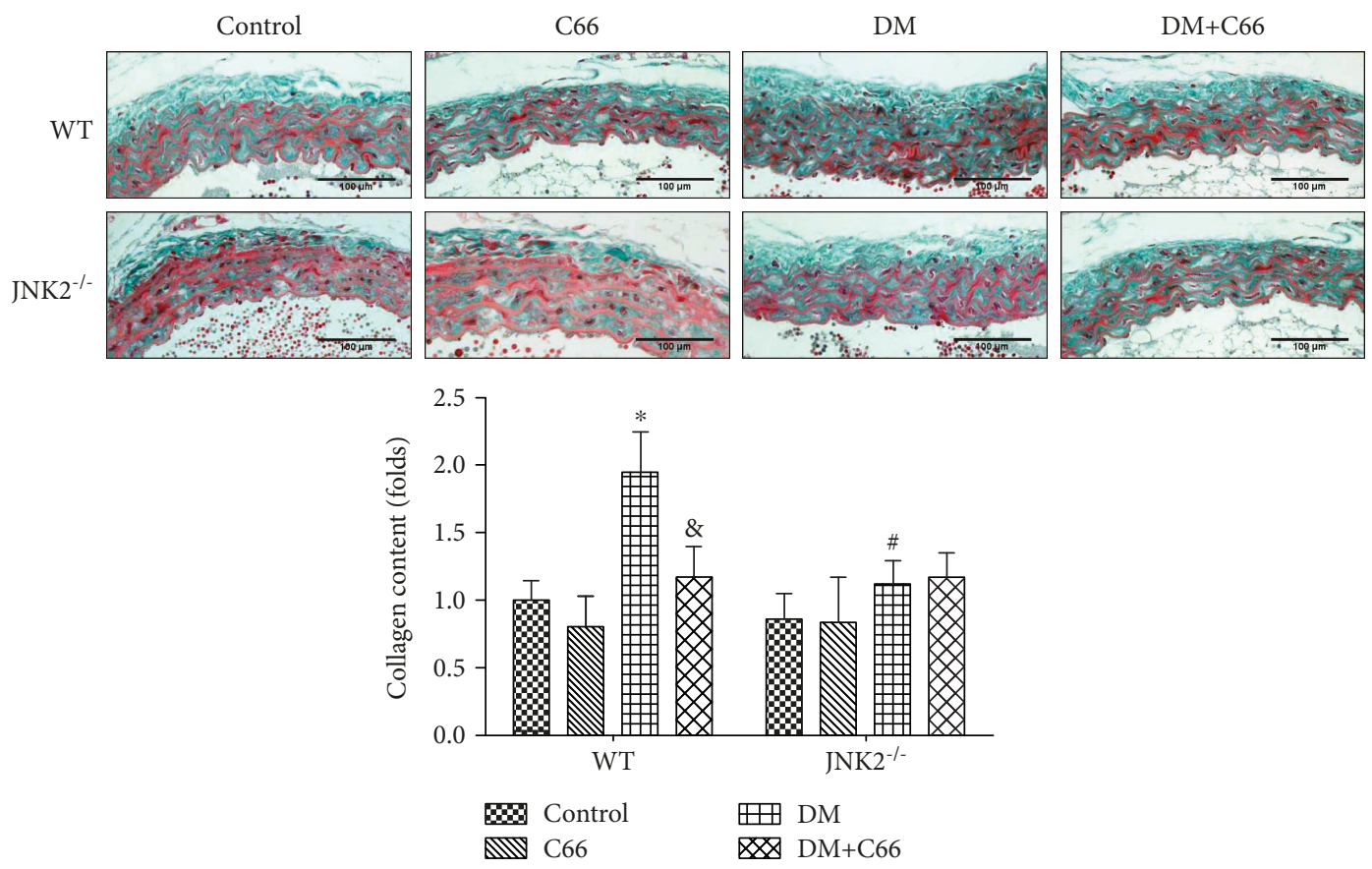

Figure 1: The accumulation of collagen was detected by Masson staining. $n=8 ;{ }^{*} P<0.05 \mathrm{DM}$ vs. corresponding control group; ${ }^{\&} P<0.05$ $\mathrm{DM}+\mathrm{C} 66$ vs. corresponding DM; ${ }^{\#} P<0.05 \mathrm{JNK}^{-1-}$ mice vs. corresponding WT mice. DM: diabetes mellitus.

C66 had no significant effect on 3-NT and 4-HNE in the aorta of JNK2 $2^{-1-}$ diabetic mice.

3.4. The Expressions of Nrf2 and Its Downstream Genes. Oxidative stress has been extensively considered as a crucial mediator for various cardiovascular complications of diabetic patients. We assumed that the above pathological changes in the aortas of diabetic mice may predominantly attribute to the increased oxidative stress. The protective effect of C66 on diabetes-induced aortic pathogenesis may be mediated by upregulation of endogenous antioxidants. Our previous study showed that C66 can upregulate the expression of Nrf2 in diabetic mice [25]. In our present study, immunofluorescent staining showed that diabetics have slightly increased expression and nuclear translocation of Nrf2, which can be significantly increased by C66 treatment or JNK2 deletion (Figures 6(a), 6(b), and 6(d)). However, C66 treatment had no extra effect on $\mathrm{Nrf} 2$ expression in JNK2 ${ }^{-1-}$ DM mice. The mRNA level of Nrf2 was consistent with immunofluorescent staining (Figure 6(c)).

The Nrf2 downstream genes SOD-1 (Figures 7(a)-7(c)) and HO-1 (Figures 7(d)-7(f)) were also evaluated by immunohistochemical staining and qPCR. The results were consistent with Nrf2.

\section{Discussion}

We have provided the first evidence to show the significant protective effect of $\mathrm{C} 66$ on diabetes-induced aortic damage. In STZ-induced WT diabetic mice, significant increase in aortic oxidative damage, inflammation, apoptosis, and fibrosis has been found. All these pathogenic changes were obviously decreased by C66 treatment or JNK2 deletion. We also demonstrated that in the JNK2 deletion DM mice, the protective effect of $\mathrm{C} 66$ in the aorta cannot be revealed. These results suggest that C66 prevents diabetes-induced pathogenic changes in the aorta via inhibition of JNK2 function.

Inflammation plays a critical role in the development of diabetes and its complications. Chronic inflammation induces oxidative stress, apoptosis, endothelial dysfunction, and fibrosis, all of which contribute to tissue damage and the formation of new vascular structures $[12,25]$. In our study, we showed the increased expressions of TNF- $\alpha$ and MCP-1 (Figure 4), as inflammation markers, in the aorta of the WT DM group, which was accompanied with increased expressions of markers of aortic fibrosis (CTGF, TGF- $\beta 1$ ) (Figure 2), apoptosis (caspase-3) (Figure 3), and oxidative stress (3-NT, 4-HNE) (Figure 5) in the WT DM group. Either C66 treatment or JNK2 deletion can eliminate these increased expressions of markers in the aortas of diabetic mice. And C66 treatment was found no further effect on the JNK2 $2^{-1-} \mathrm{DM}$ group suggesting that the protection of C66 may target on JNK2 inhibition.

JNK, as a member of the mitogen-activated protein kinase family, regulates various cell stress responses, including inflammatory responses, oxidative stress, cell death, cell survival, and proteins expression [27]. In diabetes, obvious and sustained JNK activation is observed in different tissues $[23,25,28,29]$. Thus, deregulating the activation of JNK is a potent therapeutic strategy for diabetes. Curcumin has shown to protect cardiovascular diseases via inhibition of JNK $[30,31]$. In the previous studies from our team, C66, as a novel curcumin analogue, has also been revealed significant effect on inhibition of JNK $[23,25]$. Moreover, Pan et al. have reported that C66 exhibits a high JNK2-binding affinity in a molecular docking, which leads to its anti-inflammatory 

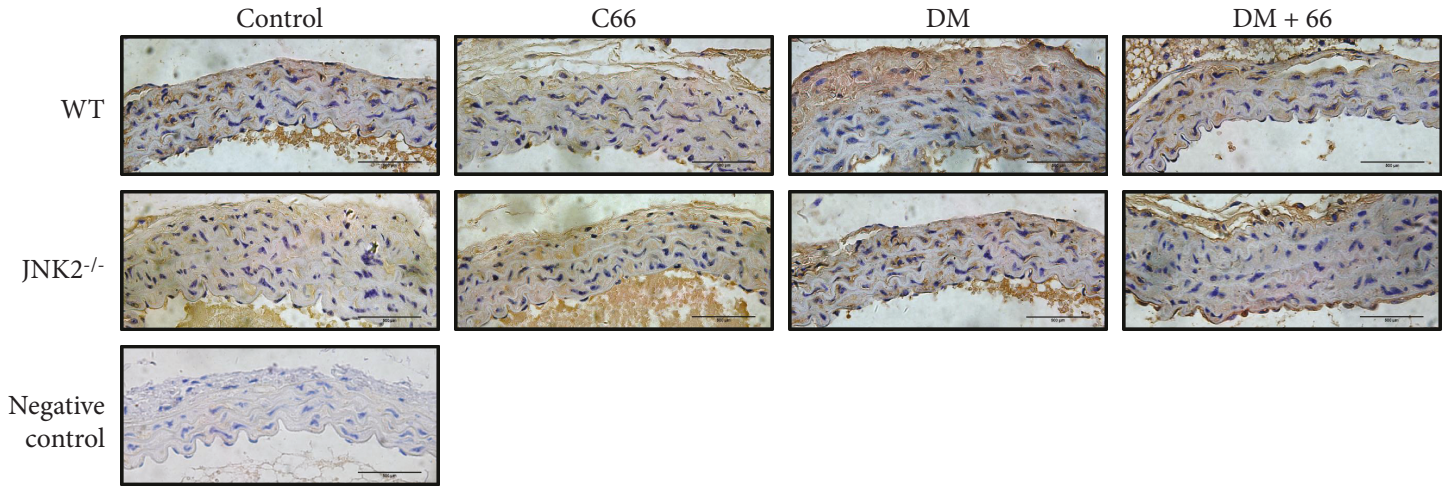

(a)
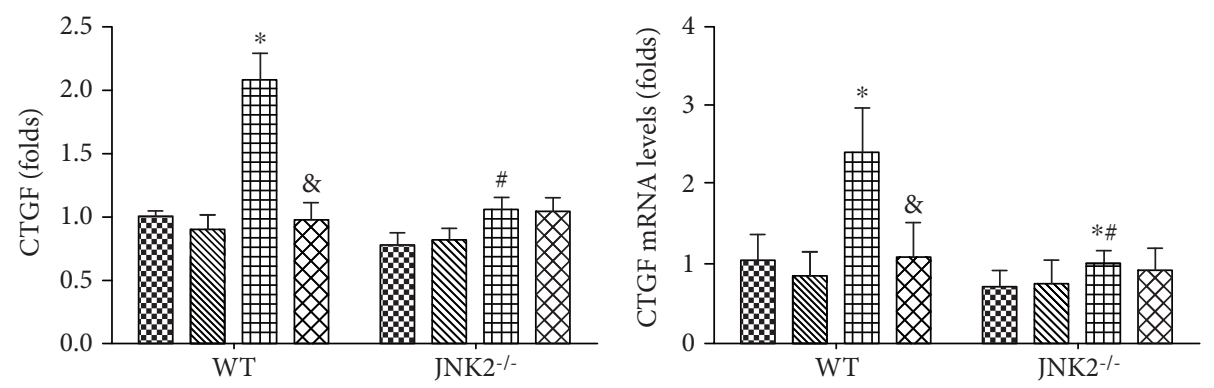

$\begin{array}{ll}\text { Control } & \text { DM } \\ \text { C66 } & \text { DM }+ \text { C66 }\end{array}$

(b)
80 Control
世田 DM
C66

(c)
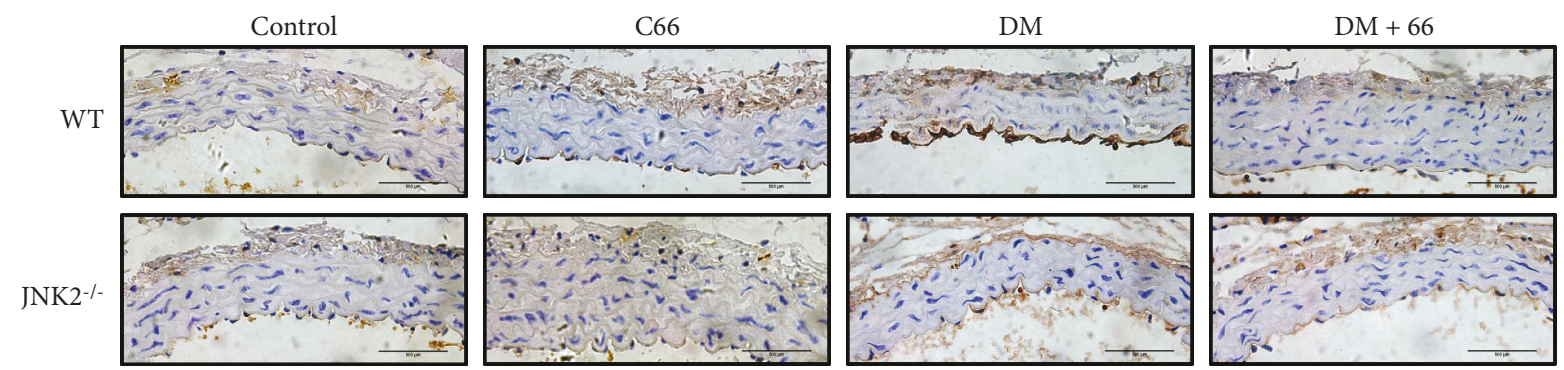

(d)

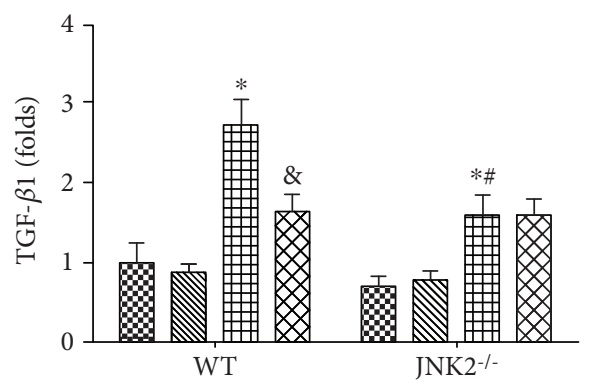

80 Contro

\# DM

C66

DM + C66

(e)

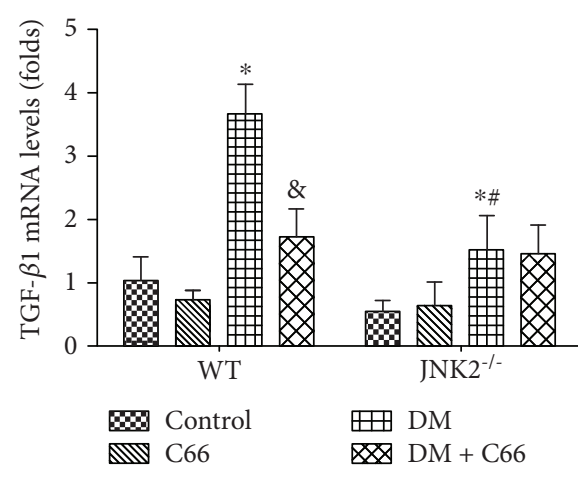

(f)

FIgURE 2: Protective effect of C66 or JNK2 deletion on diabetes-induced aortic fibrosis. Immunohistochemical staining for the expression of $\operatorname{CTGF}(\mathrm{a}, \mathrm{b})$ and TGF- $\beta 1(\mathrm{~d}, \mathrm{e})$ and qPCR for the mRNA expression of CTGF (c) and TGF- $\beta 1$ (f). $n=8$; ${ }^{*} P<0.05$ DM vs. corresponding control group; ${ }^{\&} P<0.05 \mathrm{DM}+\mathrm{C} 66$ vs. corresponding $\mathrm{DM} ;{ }^{\#} P<0.05 \mathrm{JNK} 2^{-/-}$mice vs. corresponding WT mice. DM: diabetes mellitus. 

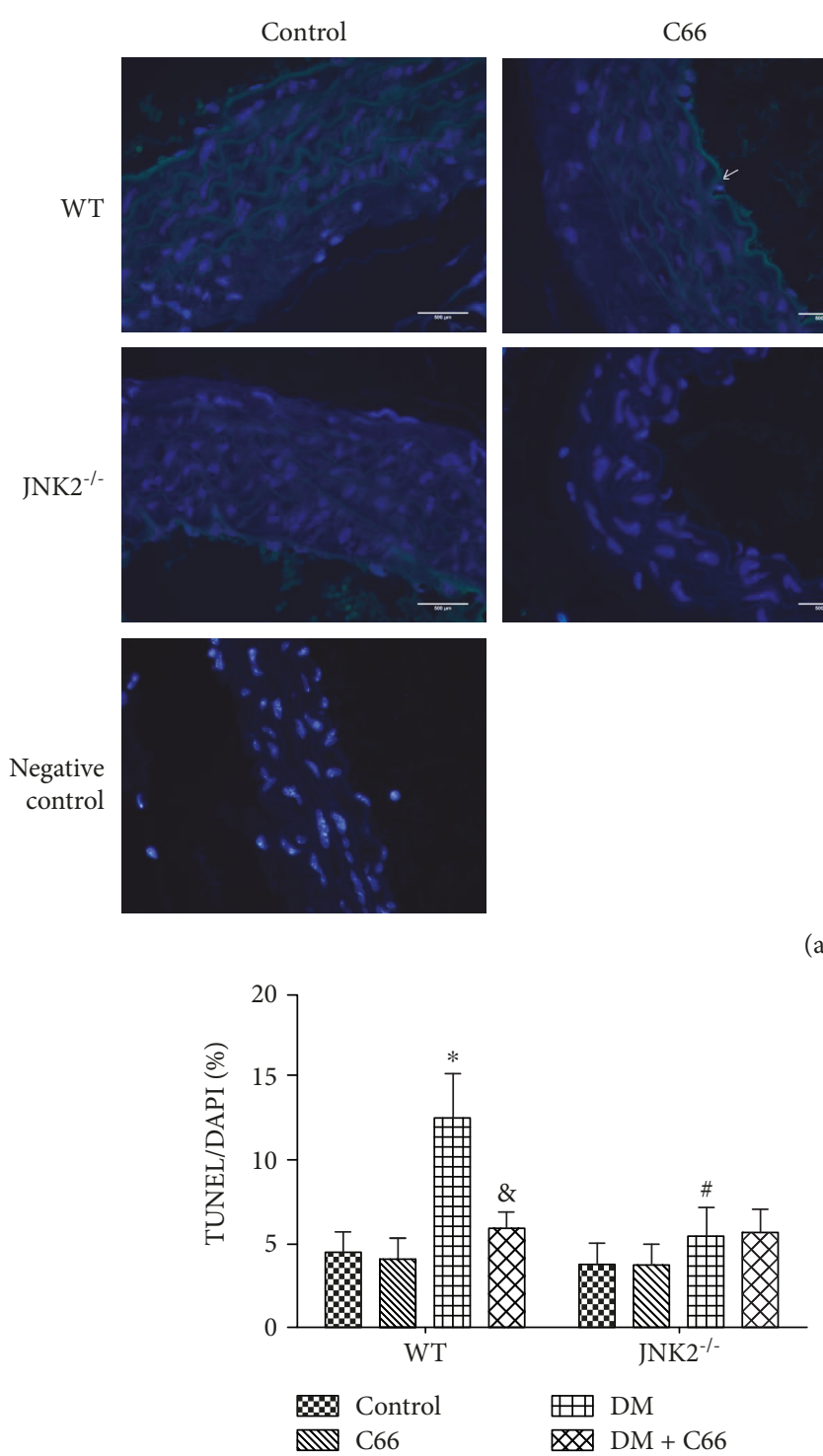

(b)
C66
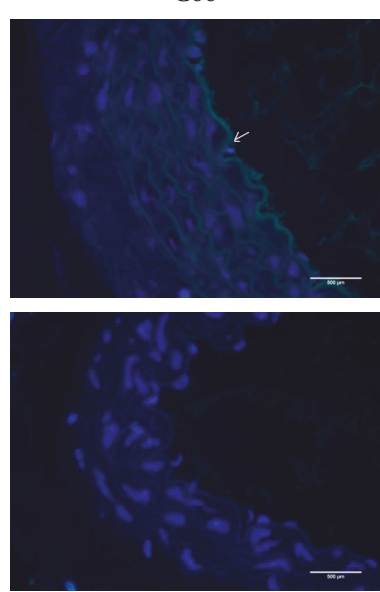

$\mathrm{DM}$
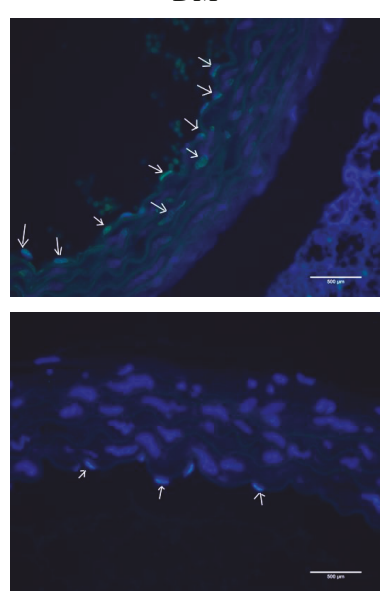

$\mathrm{DM}+\mathrm{C} 66$
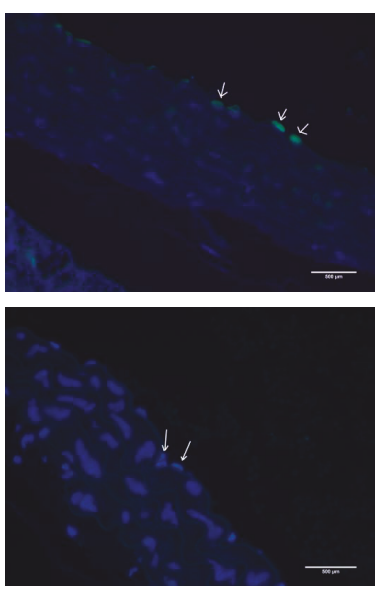

(a)

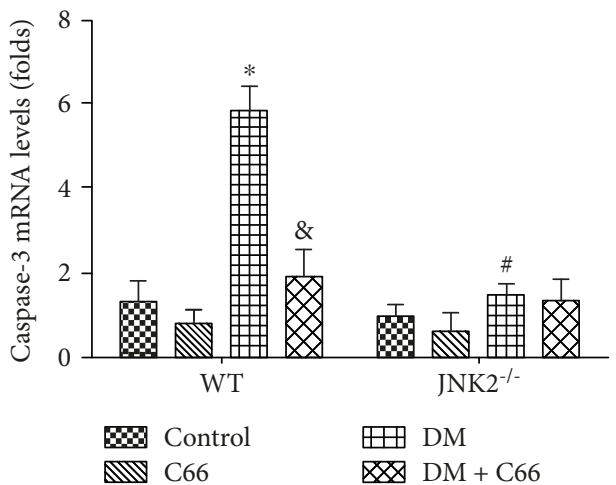

(c)

FIgURE 3: Protective effect of C66 or JNK2 deletion on diabetes-induced aortic apoptosis. The apoptotic cell was examined by TUNEL staining ( $\mathrm{a}, \mathrm{b}$ ); the expression of caspase- 3 mRNA (c) reflects the apoptotic level. $n=8 ;{ }^{*} P<0.05$ DM vs. corresponding control group; ${ }^{\&} \mathrm{P}<0.05 \mathrm{DM}+\mathrm{C} 66$ vs. corresponding DM; ${ }^{\#} \mathrm{P}<0.05 \mathrm{JNK} 2^{-/-}$mice vs. corresponding WT mice. DM: diabetes mellitus.

actions [26]. In our present study, we have demonstrated that JNK2 deletion indeed alleviated diabetes-induced aortic inflammation, oxidative stress, apoptosis, and fibrosis, but there was no obvious effect in the $\mathrm{JNK}^{-1-}$ diabetic mice aorta after $\mathrm{C} 66$ treatment. These results suggest that the protection of $\mathrm{C} 66$ on diabetes-induced aortic damage may depend on JNK2 suppression.

Oxidative stress is involved in the pathogenesis of diabetes-induced cardiovascular changes, and hyperglycemia is the causal link between diabetes and increased oxidative stress [32, 33]. Excessive ROS generation has been identified as an initial pathogenic factor of diabetic aortic damage. Hence, enhanced endogenous antioxidative capacity has been considered effective in attenuating these damages. Nrf2 regulates multiple adaptive responses to oxidative stress and is also involved in cell migration, proliferation, apoptosis, and differentiation [34]. And Nrf2 has been shown therapeutic effects in diabetic complications by contributing to the inducible expression of antioxidant enzymes. Nrf2 silencing has been verified to inhibit the migration, proliferation, and secretion of endothelial progenitor cells, but increases oxidative stress and cell senescence [35]. Furthermore, overexpression of Nrf2 inhibits ROS and inflammatory cytokine expression in the high glucose-cultured endothelial progenitor cells [36]. As a downstream factor of JNK, we speculated that Nrf2 may involve in the C66 protection. Here, we verified that diabetes can slightly increase Nrf2 expression and function, which were reflected by $\mathrm{HO}-1$ and SOD-1. It is suggested that at certain early stages, Nrf2 acts as a protective mechanism attempting to protect the tissue, which was consistent with our previous research [25]. In the present study, a mild increase in Nrf2 expression and 


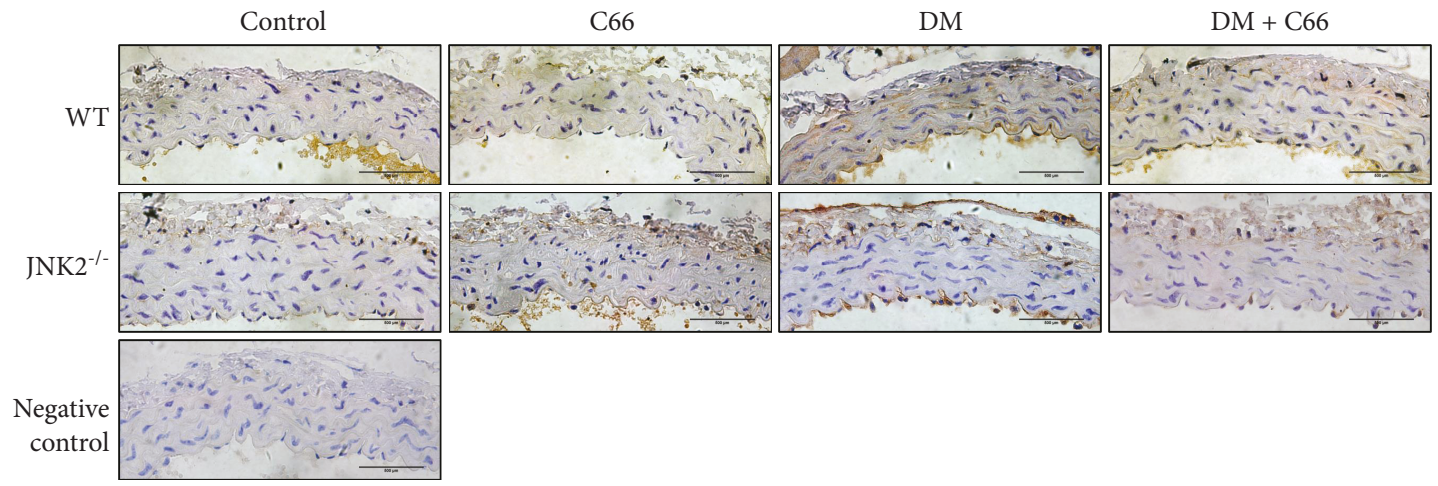

(a)

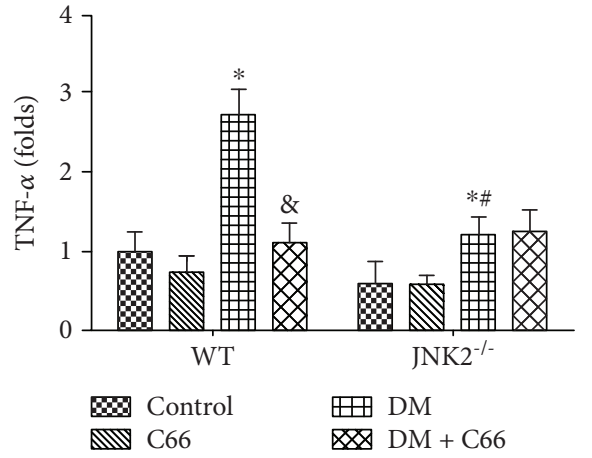

(b)

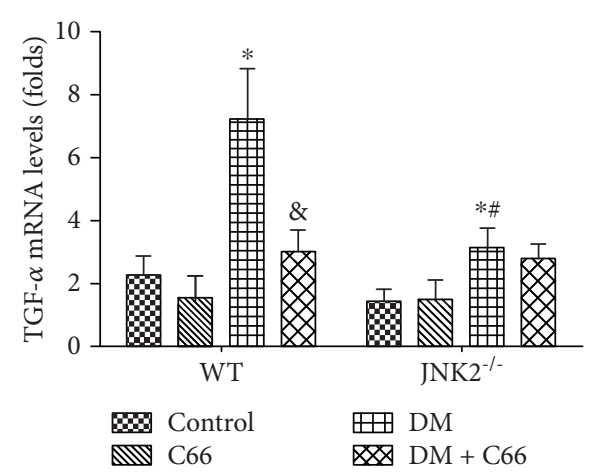

(c)
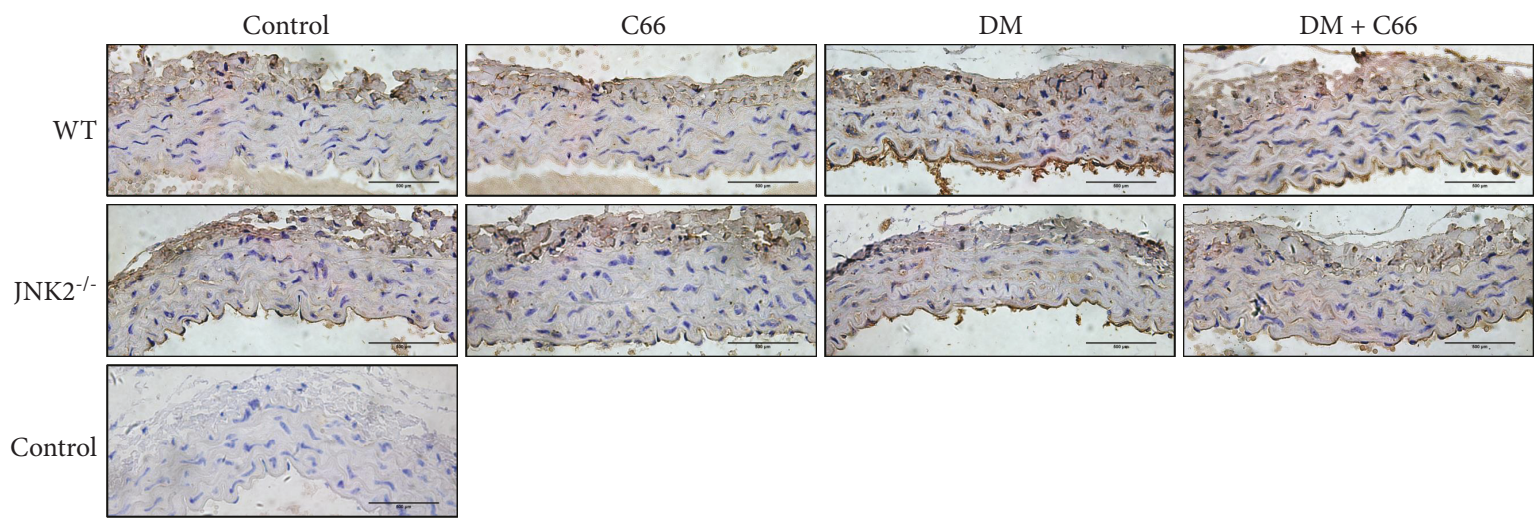

(d)

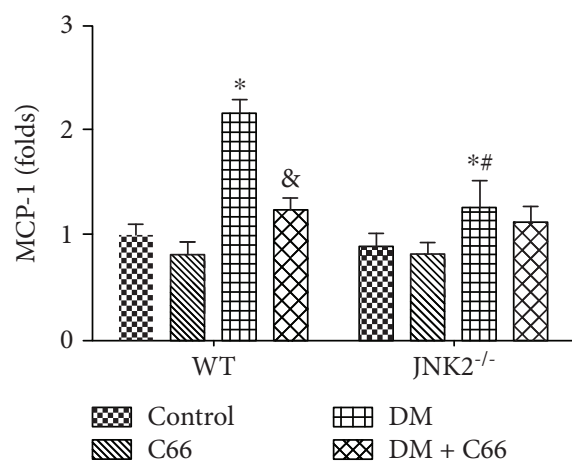

(e)

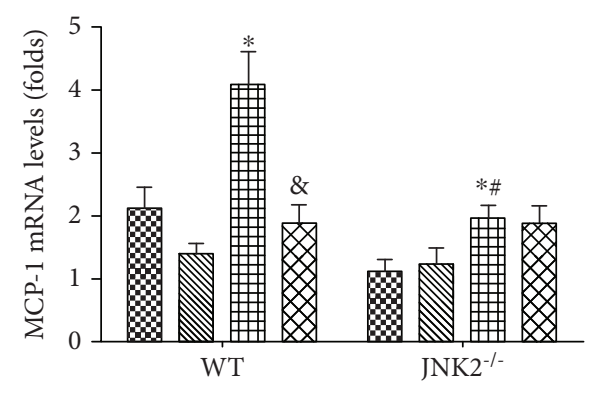

$\infty$ Control \#DM

C66 $\mathrm{DM}+\mathrm{C} 66$

(f)

Figure 4: Protective effect of C66 or JNK2 deletion on diabetes-induced aortic inflammation. Immunohistochemical staining for the expression of TNF- $\alpha(\mathrm{a}, \mathrm{b})$ and MCP-1 (d, e) and qPCR for the mRNA expression of TNF- $\alpha(\mathrm{c})$ and MCP-1 (f). $n=8 ;{ }^{*} P<0.05$ DM vs. corresponding control group; ${ }^{\&} P<0.05 \mathrm{DM}+\mathrm{C} 66$ vs. corresponding $\mathrm{DM}$; ${ }^{\#} P<0.05 \mathrm{JNK} 2^{-l-}$ mice vs. corresponding WT mice. DM: diabetes mellitus. 

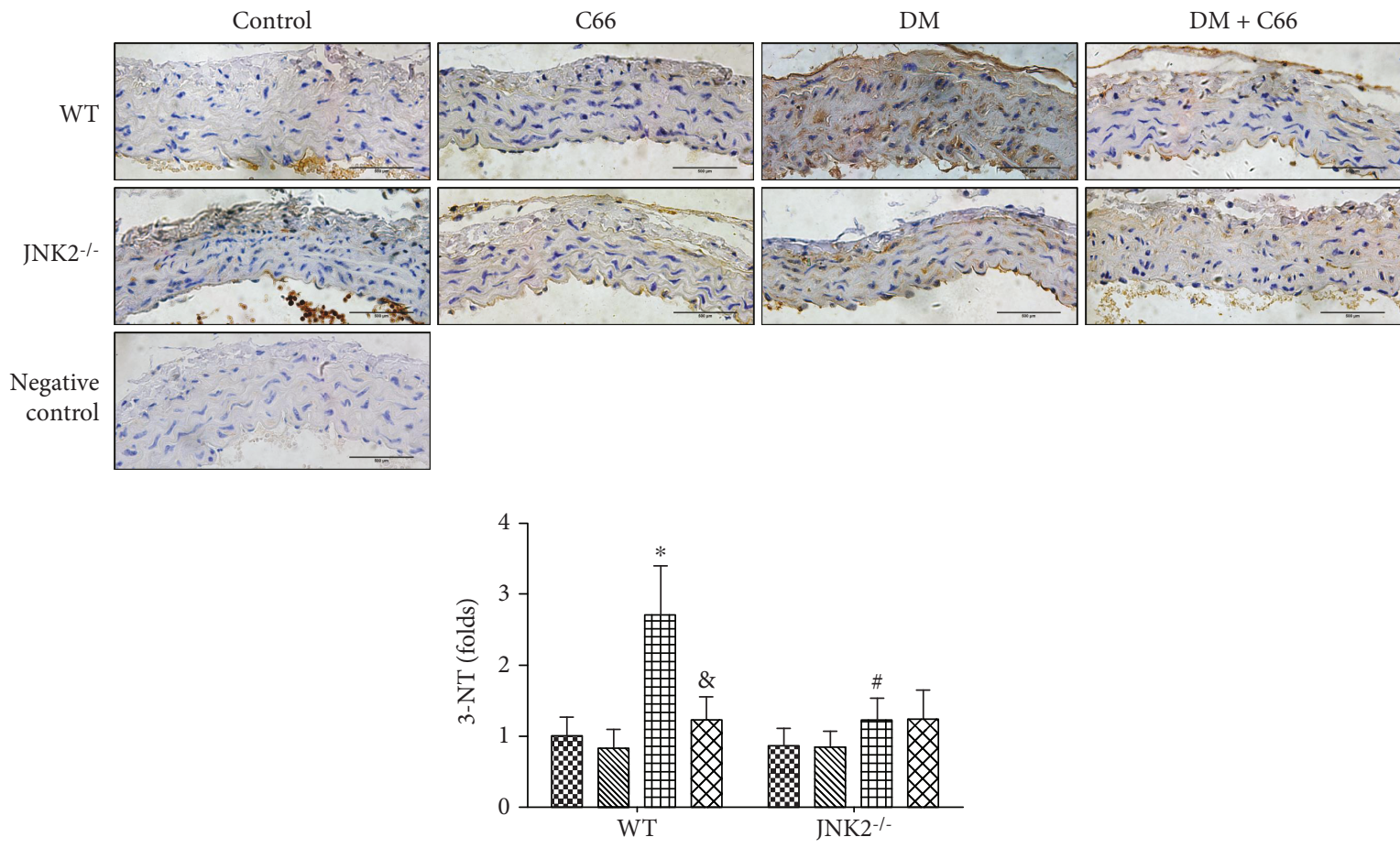

8 Control \# DM

C66 $\mathrm{DM}+\mathrm{C} 66$

(a)
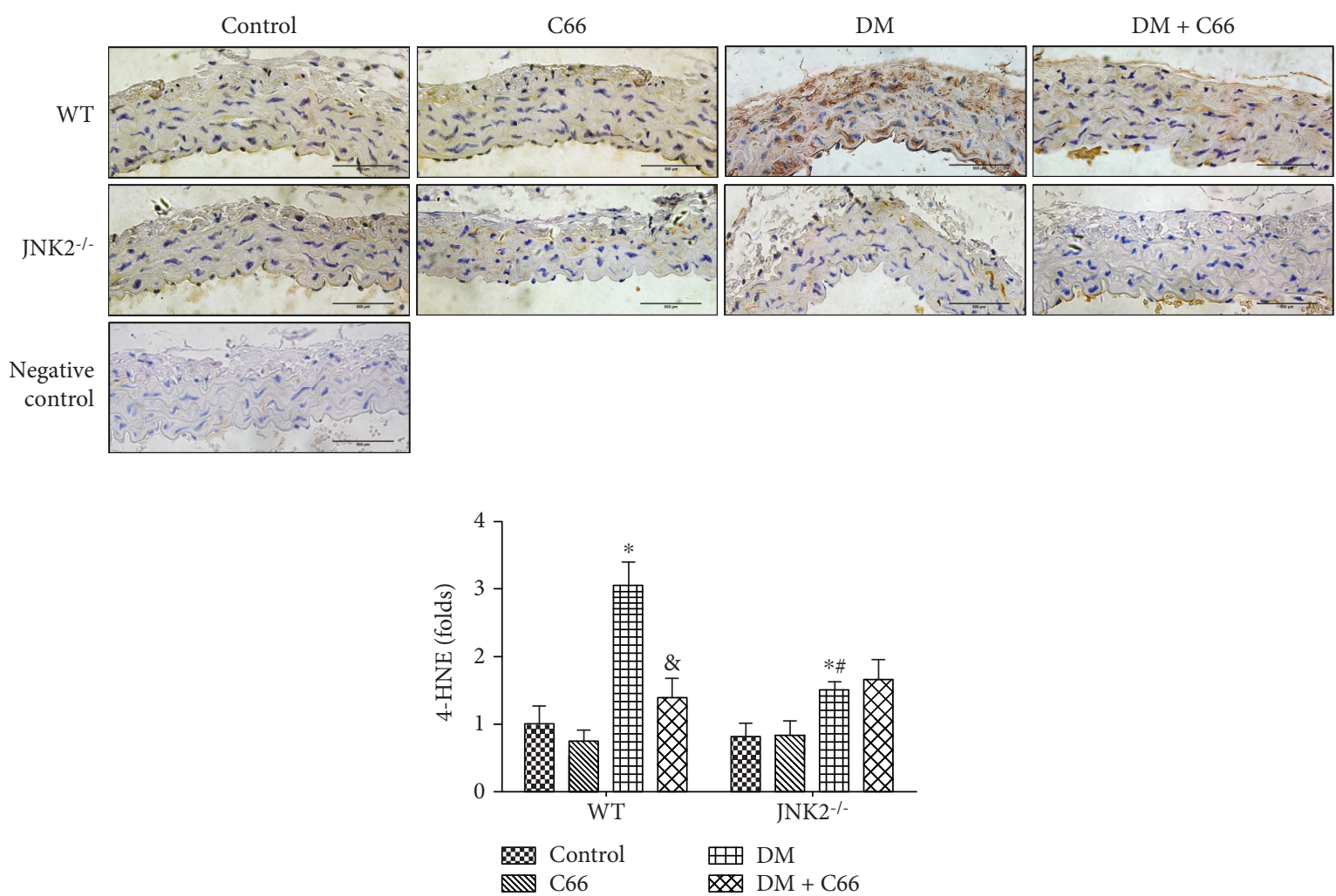

(b)

FIGURE 5: Protective effect of C66 or JNK2 deletion on diabetes-induced aortic oxidative stress. Immunohistochemical staining for the expression of $3-\mathrm{NT}$ (a) and 4 -HNE (b). $n=8 ;{ }^{*} P<0.05 \mathrm{DM}$ vs. corresponding control group; ${ }^{\mathrm{P}} \mathrm{P}<0.05 \mathrm{DM}+\mathrm{C} 66$ vs. corresponding DM; ${ }^{\#} P<0.05 \mathrm{JNK}^{-1-}$ mice vs. corresponding WT mice. DM: diabetes mellitus. 


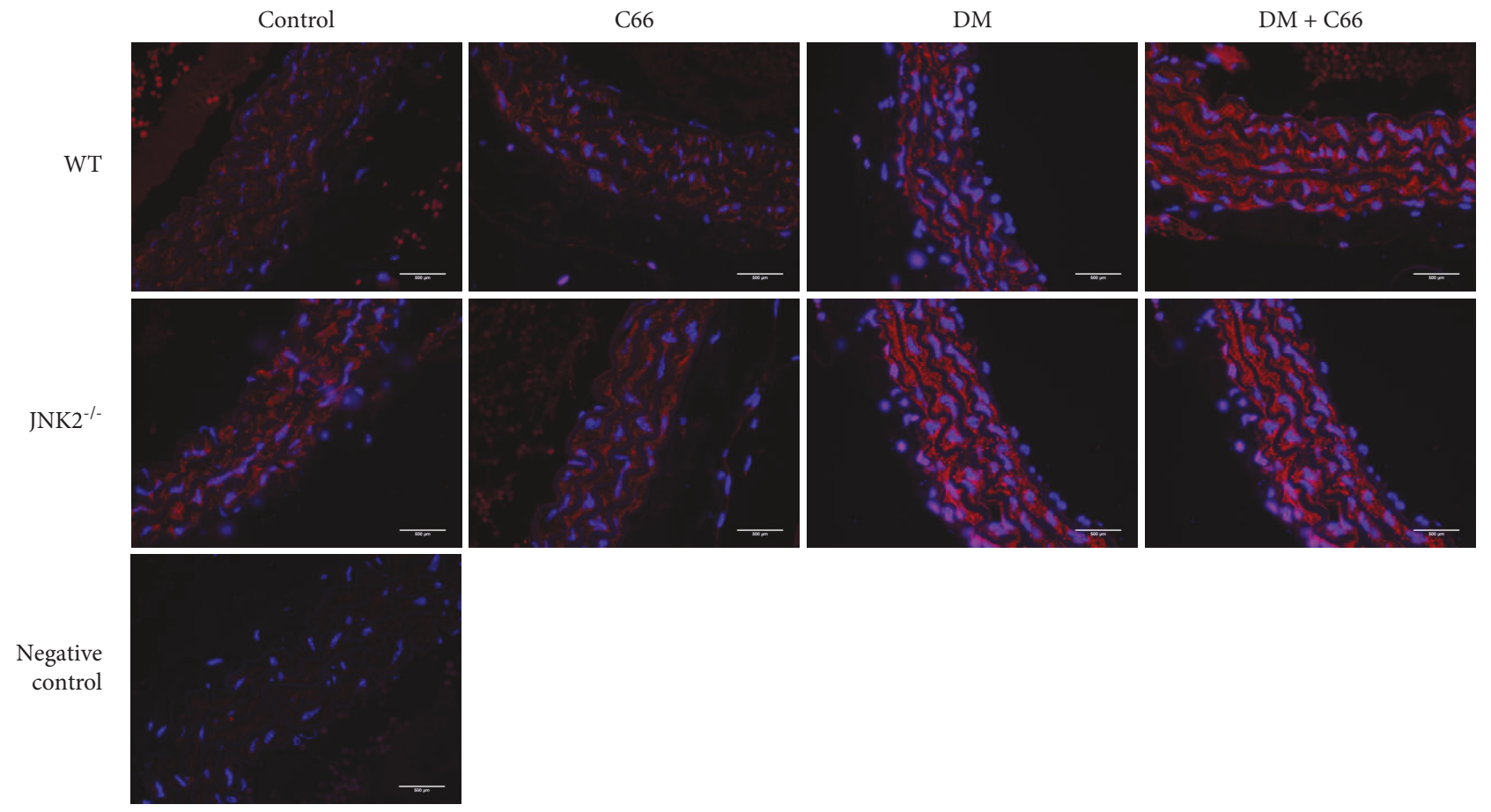

(a)

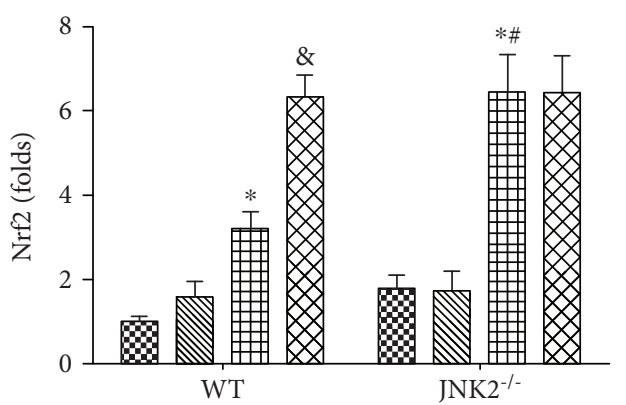

(2) Control C66
\# DM

$D M+C 66$

(b)

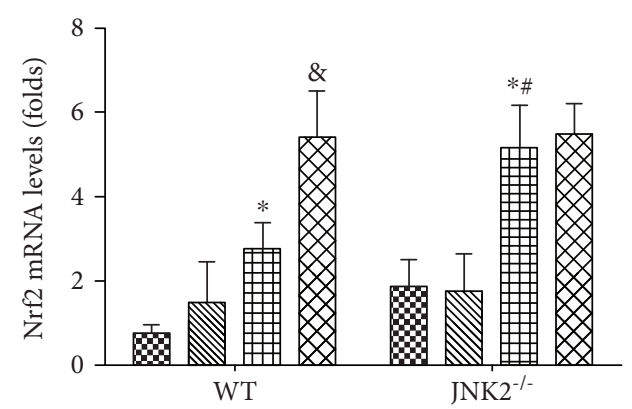

60 Control \# DM

$D M+C 66$

(c)

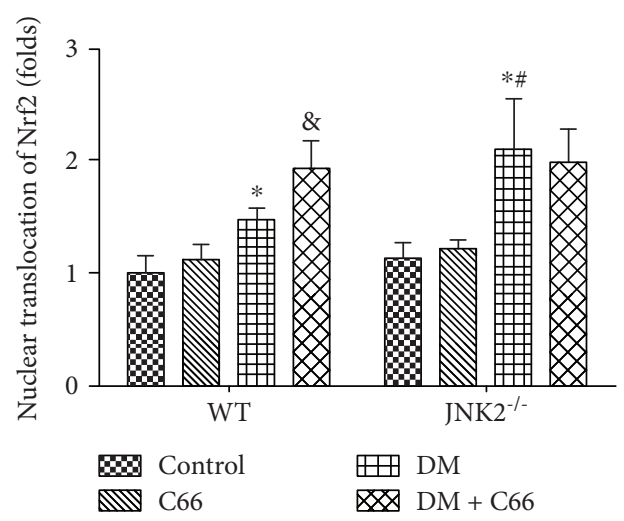

(d)

Figure 6: The expression of Nrf2 in the aortas. Aortic expression of Nrf2 was examined by immunofluorescent staining for its protein expression (red) $(\mathrm{a}, \mathrm{b})$ and real-time PCR for its mRNA level (c). The nuclear translocation of Nrf2 was also evaluated $(\mathrm{d}) . n=8$; ${ }^{*} P<0.05$ $\mathrm{DM}$ vs. corresponding control group; ${ }^{8} \mathrm{P}<0.05 \mathrm{DM}+\mathrm{C} 66$ vs. corresponding DM; ${ }^{\#} P<0.05 \mathrm{JNK} 2^{-/-}$mice vs. corresponding WT mice. DM: diabetes mellitus. 

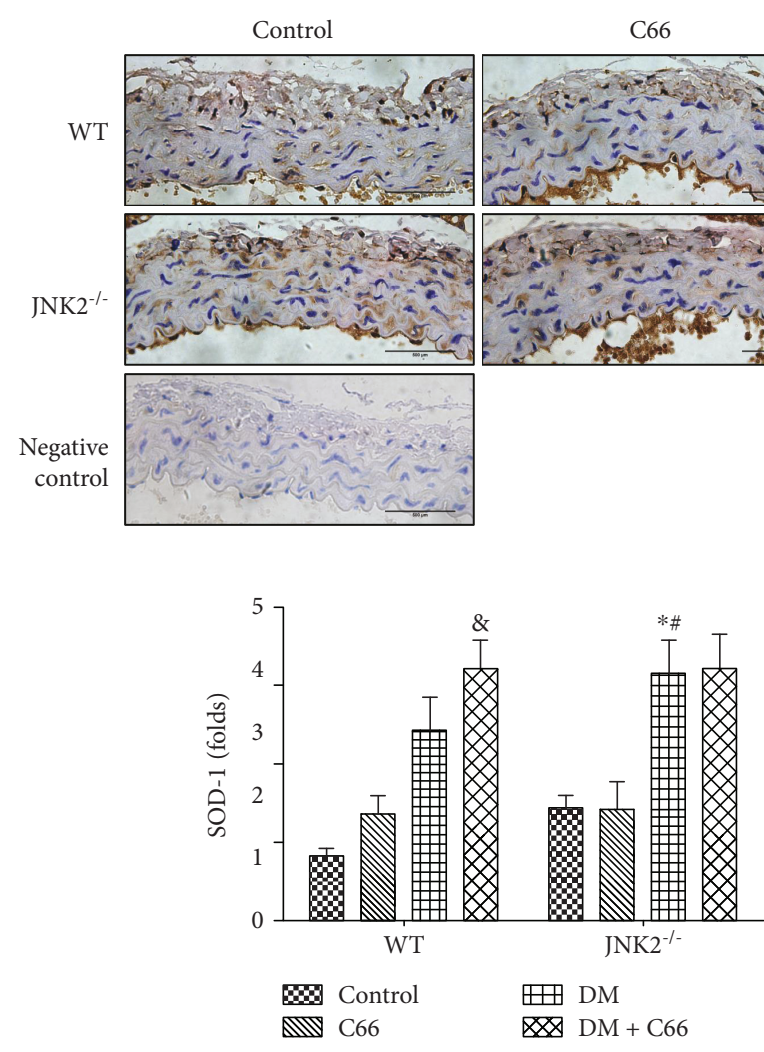

(b)
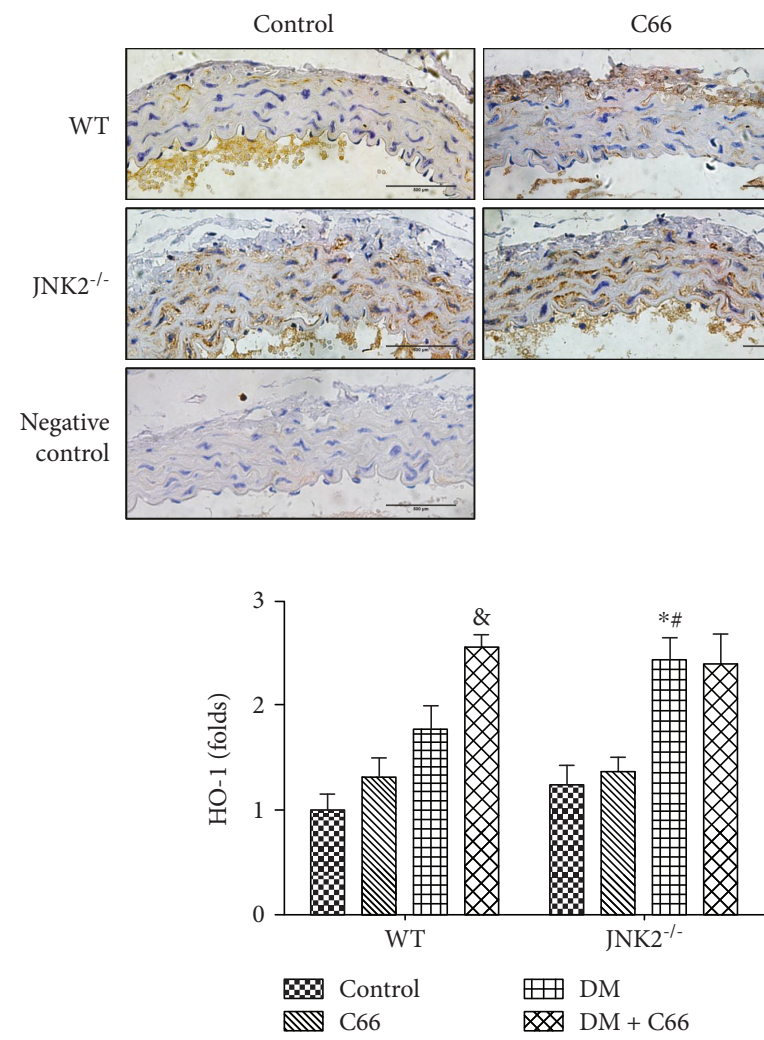

(e)
$\mathrm{DM}$

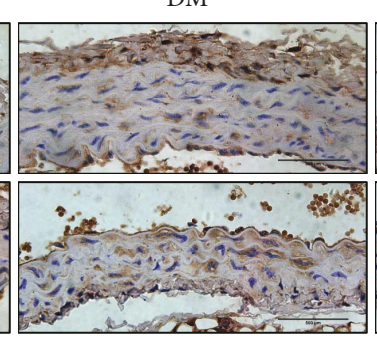

(a)

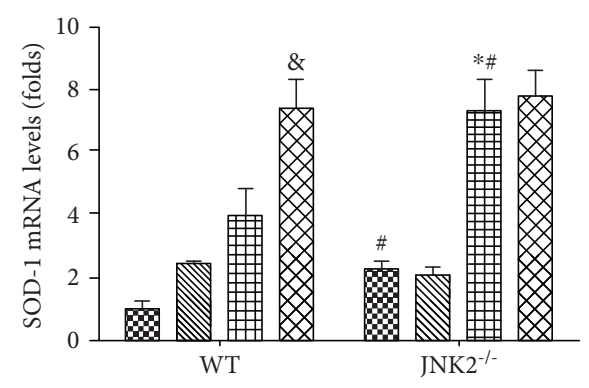

\% Control 田 DM C66

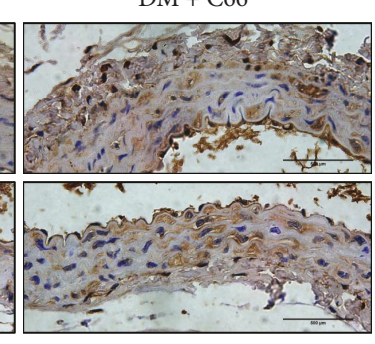

(c)

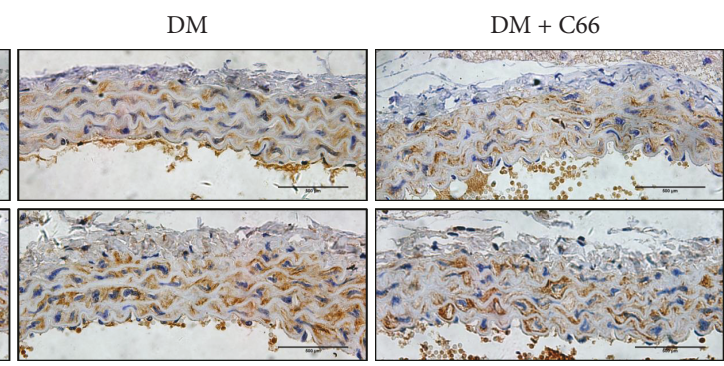

(d)

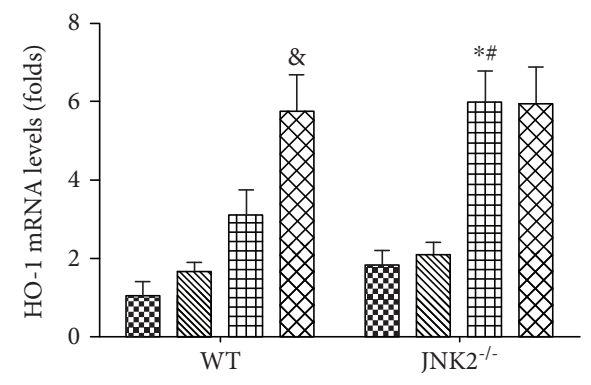

80 Control \# DM

$\mathrm{C} 66$

$\mathrm{DM}+\mathrm{C} 66$

(f)

FIGURE 7: The expression of Nrf2 downstream genes. Immunohistochemical staining for the expression of SOD-1 (a, b) and HO-1 (d, e) and qPCR for the mRNA expression of SOD-1 (c) and HO-1 (f). $n=8 ;{ }^{*} P<0.05$ DM vs. corresponding control group; ${ }^{8} P<0.05$ DM + C66 vs. corresponding DM; ${ }^{\#} P<0.05 \mathrm{JNK} 2^{-1-}$ mice vs. corresponding WT mice. DM: diabetes mellitus. 
function may remain not enough to compensate the severe damage induced by diabetes. Either C66 treatment or JNK2 deletion effectively increased Nrf2 expression and function (Figures 6 and 7). However, the JNK2 ${ }^{-1-} \mathrm{DM}+\mathrm{C} 66$ group has not shown significant increase of Nrf2 expression and its function, compared with the $\mathrm{JNK} 2^{-1-} \mathrm{DM}$ group. It indicated that JNK2 may be the key factor that regulates Nrf2 and its function in the protection of C66.

In conclusion, we have investigated that the protective effect of C66 in the diabetic aorta mainly depends on JNK2 inactivation. Either C66 treatment or JNK2 deletion can reverse and/or prevent the progression of diabetes-induced aortic inflammation, oxidative damage, apoptosis, and fibrosis. Mechanism responsible for this protective effect of C66 is mediated by inhibition of JNK2 that may be related to upregulation of Nrf2 expression and function.

\section{Data Availability}

The data used to support the findings of this study are included within the article.

\section{Conflicts of Interest}

No conflicts of interest, financial or otherwise, are declared by the authors.

\section{Authors' Contributions}

Cheng Li and Xiao Miao equally contributed to the work.

\section{Acknowledgments}

The authors would like to thank Professor Guang Liang and his team for providing C66 and technical support. This work was supported by the National Natural Science Foundation of China (YGW, grant number 81400279), (SDW, grant number 81770375), (JS, grant number 81770374), and (QL, grant number 81670343) and the Jilin Province Science and Technology Project (YGW, grant number 20170520012J).

\section{Supplementary Materials}

Supplementary Figure 1. The blood glucose level of each group at the beginning of the experiment. $n=8 ;{ }^{*} P<0.05$ vs. corresponding control group. Supplementary Figure 2. The hematoxylin and eosin staining of the aortas. And the wall intima thickness was evaluated. $n=8 ;{ }^{*} P<0.05$ vs. corresponding control group. (Supplementary materials)

\section{References}

[1] R. Dhingra and R. S. Vasan, "Diabetes and the risk of heart failure," Heart Failure Clinics, vol. 8, no. 1, pp. 125-133, 2012.

[2] A. Dinesh Shah, C. Langenberg, E. Rapsomaniki et al., "Type 2 diabetes and incidence of a wide range of cardiovascular diseases: a cohort study in 1.9 million people," The Lancet, vol. 385, p. S86, 2015.

[3] L. Guariguata, D. R. Whiting, I. Hambleton, J. Beagley, U. Linnenkamp, and J. E. Shaw, "Global estimates of diabetes prevalence for 2013 and projections for 2035," Diabetes Research and Clinical Practice, vol. 103, no. 2, pp. 137-149, 2014.

[4] S. Dogné, B. Flamion, and N. Caron, "Endothelial glycocalyx as a shield against diabetic vascular complications: involvement of hyaluronan and hyaluronidases," Arteriosclerosis, Thrombosis, and Vascular Biology, vol. 38, no. 7, pp. 14271439, 2018.

[5] N. K. P. Wong, S. J. Nicholls, J. T. M. Tan, and C. A. Bursill, "The role of high-density lipoproteins in diabetes and its vascular complications," International Journal of Molecular Sciences, vol. 19, no. 6, article 1680, 2018.

[6] S. M. Son, "Reactive oxygen and nitrogen species in pathogenesis of vascular complications of diabetes," Diabetes and Metabolism Journal, vol. 36, no. 3, pp. 190-198, 2012.

[7] C. Li, X. Miao, F. Li et al., "Oxidative stress-related mechanisms and antioxidant therapy in diabetic retinopathy," Oxidative Medicine and Cellular Longevity, vol. 2017, Article ID 9702820, 15 pages, 2017.

[8] D. Tousoulis, A. M. Kampoli, C. Tentolouris, N. Papageorgiou, and C. Stefanadis, "The role of nitric oxide on endothelial function," Current Vascular Pharmacology, vol. 10, no. 1, pp. 4-18, 2012.

[9] G. R. Drummond, S. Selemidis, K. K. Griendling, and C. G. Sobey, "Combating oxidative stress in vascular disease: NADPH oxidases as therapeutic targets," Nature Reviews Drug Discovery, vol. 10, no. 6, pp. 453-471, 2011.

[10] F. Paneni, J. A. Beckman, M. A. Creager, and F. Cosentino, "Diabetes and vascular disease: pathophysiology, clinical consequences, and medical therapy: part I," European Heart Journal, vol. 34, no. 31, pp. 2436-2443, 2013.

[11] L. Rochette, M. Zeller, Y. Cottin, and C. Vergely, "Diabetes, oxidative stress and therapeutic strategies," Biochimica et Biophysica Acta, vol. 1840, no. 9, pp. 2709-2729, 2014.

[12] Y. Wang, Z. Zhang, W. Sun et al., "Sulforaphane attenuation of type 2 diabetes-induced aortic damage was associated with the upregulation of Nrf2 expression and function," Oxidative Medicine and Cellular Longevity, vol. 2014, Article ID 123963, 11 pages, 2014.

[13] D. V. Chartoumpekis, D. L. Palliyaguru, N. Wakabayashi et al., "Nrf2 deletion from adipocytes, but not hepatocytes, potentiates systemic metabolic dysfunction after long-term high-fat diet-induced obesity in mice," American Journal of Physiology-Endocrinology and Metabolism, vol. 315, no. 2, pp. E180-E195, 2018.

[14] M. Pal, M. A. Febbraio, and G. I. Lancaster, "The roles of c-Jun NH2-terminal kinases (JNKs) in obesity and insulin resistance," The Journal of Physiology, vol. 594, no. 2, pp. 267-279, 2016.

[15] R. J. Davis, "Signal transduction by the JNK group of MAP kinases," Cell, vol. 103, no. 2, pp. 239-252, 2000.

[16] E. Osto, C. M. Matter, A. Kouroedov et al., "c-Jun N-terminal kinase 2 deficiency protects against hypercholesterolemiainduced endothelial dysfunction and oxidative stress," Circulation, vol. 118, no. 20, pp. 2073-2080, 2008.

[17] R. Ricci, G. Sumara, I. Sumara et al., "Requirement of JNK2 for scavenger receptor A-mediated foam cell formation in atherogenesis," Science, vol. 306, no. 5701, pp. 1558-1561, 2004.

[18] G. Tuncman, J. Hirosumi, G. Solinas, L. Chang, M. Karin, and G. S. Hotamisligil, "Functional in vivo interactions between JNK1 and JNK2 isoforms in obesity and insulin resistance," 
Proceedings of the National Academy of Sciences of the United States of America, vol. 103, no. 28, pp. 10741-10746, 2006.

[19] P. Yang, Y. Cao, and H. Li, "Hyperglycemia induces inducible nitric oxide synthase gene expression and consequent nitrosative stress via c-Jun N-terminal kinase activation," American Journal of Obstetrics and Gynecology, vol. 203, no. 2, pp. 185.e5-185.e11, 2010.

[20] M. E. Wang, Y. C. Chen, I. S. Chen, S. C. Hsieh, S. S. Chen, and C. H. Chiu, "Curcumin protects against thioacetamideinduced hepatic fibrosis by attenuating the inflammatory response and inducing apoptosis of damaged hepatocytes," The Journal of Nutritional Biochemistry, vol. 23, no. 10, pp. 1352-1366, 2012.

[21] J. Zheng, J. Cheng, S. Zheng, Q. Feng, and X. Xiao, "Curcumin, a polyphenolic curcuminoid with its protective effects and molecular mechanisms in diabetes and diabetic cardiomyopathy," Frontiers in Pharmacology, vol. 9, p. 472, 2018.

[22] R. De, P. Kundu, S. Swarnakar et al., "Antimicrobial activity of curcumin against Helicobacter pylori isolates from India and during infections in mice," Antimicrobial Agents and Chemotherapy, vol. 53, no. 4, pp. 1592-1597, 2009.

[23] Y. Wang, S. Zhou, W. Sun et al., "Inhibition of JNK by novel curcumin analog C66 prevents diabetic cardiomyopathy with a preservation of cardiac metallothionein expression," American Journal of Physiology-Endocrinology and Metabolism, vol. 306, no. 11, pp. E1239-E1247, 2014.

[24] Y. Pan, Y. Wang, Y. Zhao et al., "Inhibition of JNK phosphorylation by a novel curcumin analog prevents high glucoseinduced inflammation and apoptosis in cardiomyocytes and the development of diabetic cardiomyopathy," Diabetes, vol. 63, no. 10, pp. 3497-3511, 2014.

[25] Y. Liu, Y. Wang, X. Miao et al., "Inhibition of JNK by compound C66 prevents pathological changes of the aorta in STZ-induced diabetes," Journal of Cellular and Molecular Medicine, vol. 18, no. 6, pp. 1203-1212, 2014.

[26] Y. Pan, X. Zhang, Y. Wang et al., "Targeting JNK by a new curcumin analog to inhibit NF-kB-mediated expression of cell adhesion molecules attenuates renal macrophage infiltration and injury in diabetic mice," PLoS One, vol. 8, no. 11, article e79084, 2013.

[27] G. Solinas and B. Becattini, "JNK at the crossroad of obesity, insulin resistance, and cell stress response," Molecular Metabolism, vol. 6, no. 2, pp. 174-184, 2017.

[28] J. Zhou, X. du, M. Long et al., "Neuroprotective effect of berberine is mediated by MAPK signaling pathway in experimental diabetic neuropathy in rats," European Journal of Pharmacology, vol. 774, pp. 87-94, 2016.

[29] Z. Jiao, J. Chen, Y. Liu, T. Liu, K. Chen, and G. Li, "Role of ERK1/2 and JNK phosphorylation in iodine contrast agentinduced apoptosis in diabetic rat kidneys," Renal Failure, vol. 37, no. 8, pp. 1349-1355, 2015.

[30] J. Fan, X. Li, Y. W. Yan et al., "Curcumin attenuates rat thoracic aortic aneurysm formation by inhibition of the c-Jun N-terminal kinase pathway and apoptosis," Nutrition, vol. 28, no. 10, pp. 1068-1074, 2012.

[31] C. Fiorillo, M. Becatti, A. Pensalfini et al., "Curcumin protects cardiac cells against ischemia-reperfusion injury: effects on oxidative stress, NF- $\kappa \mathrm{B}$, and JNK pathways," Free Radical Biology and Medicine, vol. 45, no. 6, pp. 839-846, 2008.

[32] A. C. Maritim, R. A. Sanders, and J. B. Watkins, "Diabetes, oxidative stress, and antioxidants: a review," Journal of
Biochemical and Molecular Toxicology, vol. 17, no. 1, pp. 2438, 2003.

[33] T. V. Fiorentino, A. Prioletta, P. Zuo, and F. Folli, "Hyperglycemia-induced oxidative stress and its role in diabetes mellitus related cardiovascular diseases," Current Pharmaceutical Design, vol. 19, no. 32, pp. 5695-5703, 2013.

[34] T. W. Kensler, N. Wakabayashi, and S. Biswal, "Cell survival responses to environmental stresses via the Keap1-Nrf2-ARE pathway," Annual Review of Pharmacology and Toxicology, vol. 47, no. 1, pp. 89-116, 2007.

[35] R.-Y. Wang, L.-H. Liu, H. Liu et al., "Nrf2 protects against diabetic dysfunction of endothelial progenitor cells via regulating cell senescence," International Journal of Molecular Medicine, vol. 42, no. 3, pp. 1327-1340, 2018.

[36] X. Li, X. Xie, W. Lian et al., "Exosomes from adipose-derived stem cells overexpressing Nrf2 accelerate cutaneous wound healing by promoting vascularization in a diabetic foot ulcer rat model," Experimental \& Molecular Medicine, vol. 50, no. 4, p. 29, 2018. 


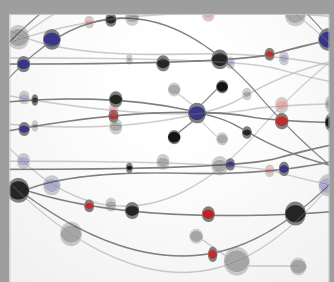

The Scientific World Journal
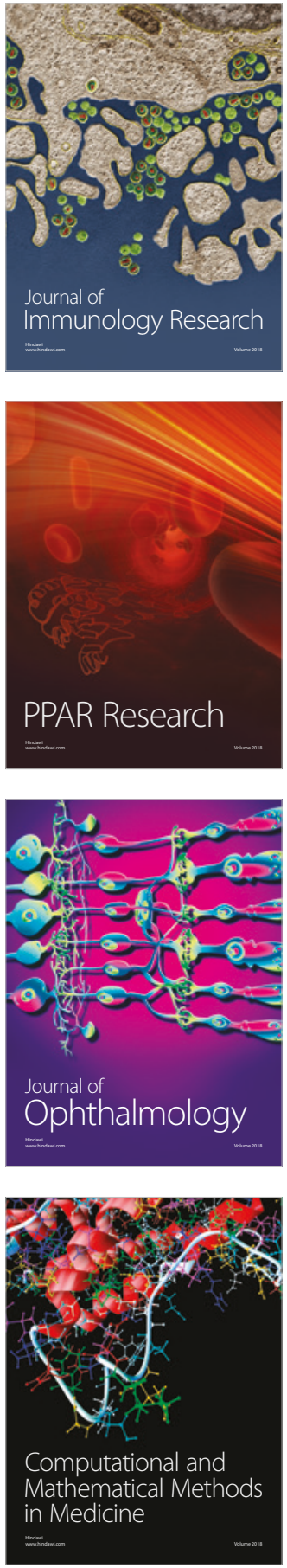

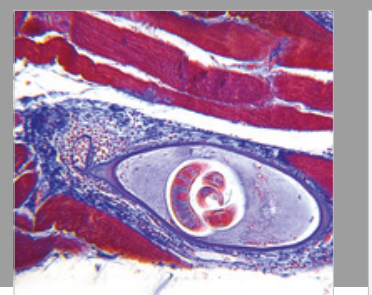

Gastroenterology Research and Practice

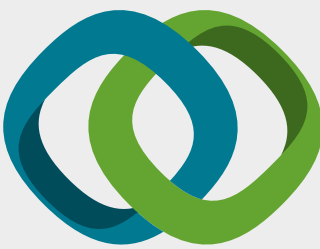

\section{Hindawi}

Submit your manuscripts at

www.hindawi.com
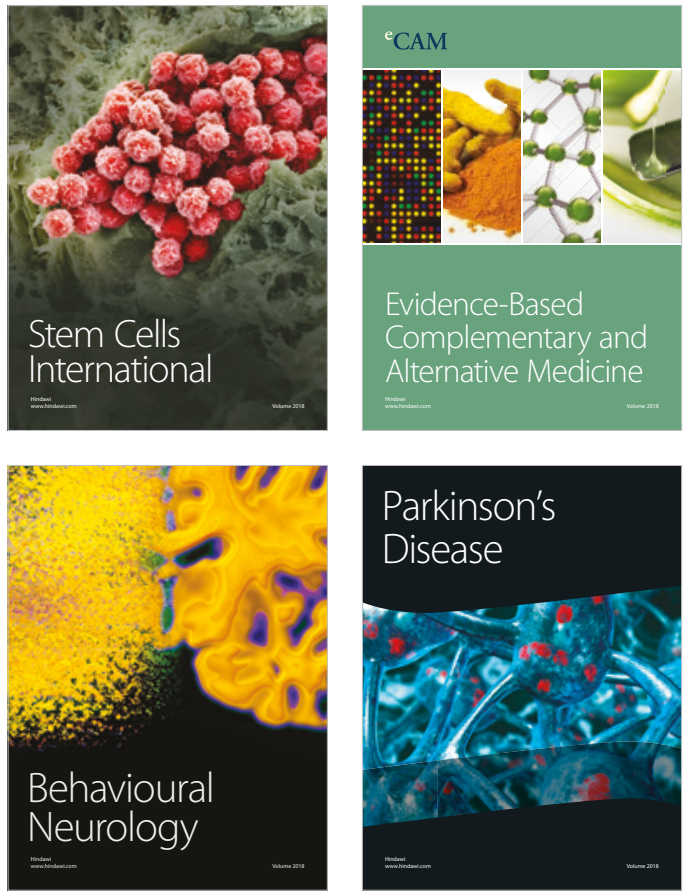

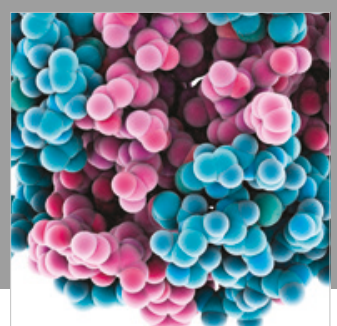

ournal of

Diabetes Research

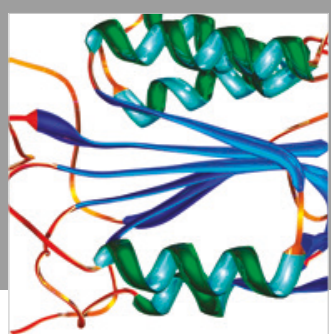

Disease Markers
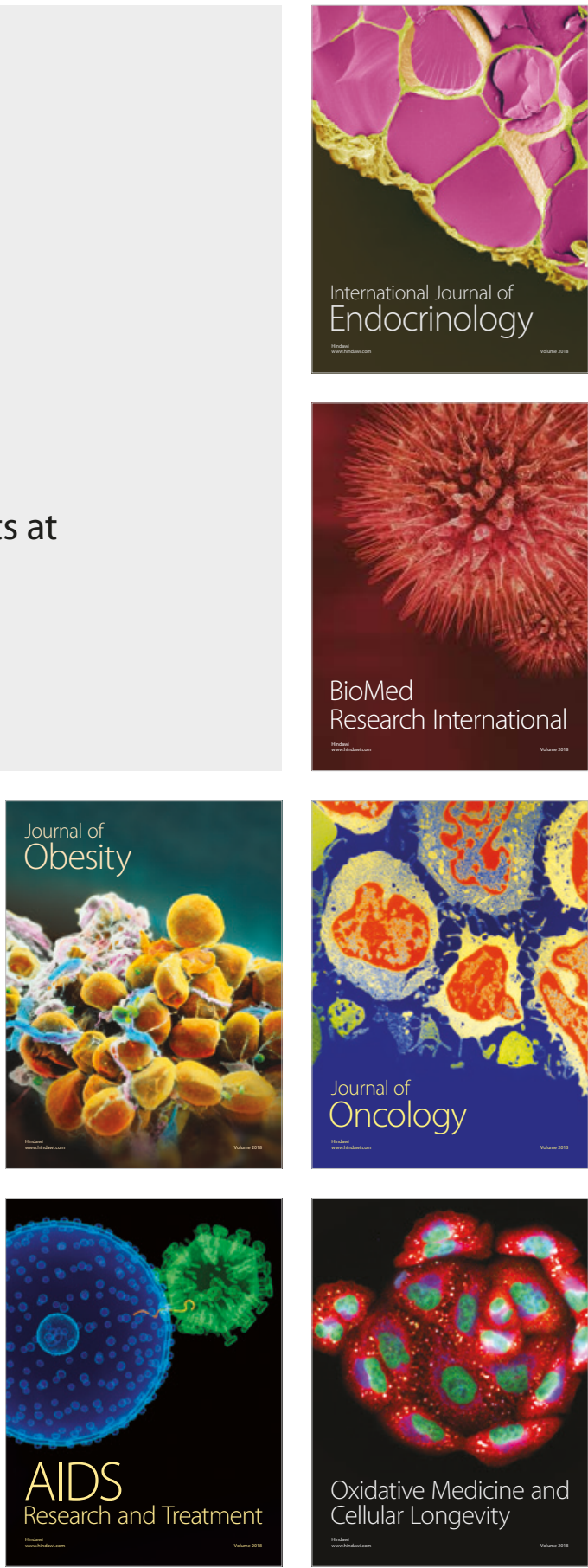